\title{
Preface - Groundwater and microbial processes
}

\author{
Barbara Bekins, Guest Editor
}

The subsurface below the soil zone harbors a large but poorly understood variety of microorganisms whose activities are important to both geologic processes and human endeavors. With the expansion of interest in this field, new techniques and strategies have been formulated to facilitate studies of subsurface microbial processes. These include collection of uncontaminated deep cores, analyses of microbial biomarkers, and interdisciplinary collaborations between hydrogeologists, geochemists, and microbiologists. These techniques are beginning to provide some insights into the subsurface biosphere.

The guiding philosophy in organizing this theme issue on "Groundwater and Microbial Processes" has been to provide an overview of the current state of this rapidly changing field. The articles are organized around three main themes: populations of microorganisms in the subsurface; the role of subsurface microorganisms in controlling chemical reactions; and methods of studying subsurface microorganisms.

The discovery of viable populations of microorganisms in the subsurface at unexpected locations has been one of the major findings at the close of this century. Documentation of microbial activity at increasingly deeper, hotter, and nutrient-limited locations challenges current understanding of survival mechanisms, maintenance energy, and growth rates. The article by Krumholz provides an overview of the strategies used by microorganisms to survive in the deep subsurface. With more than two-thirds of the planet covered by oceans, the subsurface below the seafloor comprises an enormous volume of sediments and oceanic crust that is now recognized as an important microbial habitat. Parkes and his colleagues provide a summary of recent observations of bacterial populations and activities below the seafloor. Similar to the surprising survival of microbial populations in extreme subsurface conditions, the persistence and transport of viable pathogenic microorganisms in shallow aquifers used for drinking water is also greater

Barbara Bekins

U.S. Geological Survey, MS 496, 345 Middlefield Rd.,

Menlo Park, CA 94025

Fax: +1 650-329-5590

e-mail: babekins@usgs.gov than previously thought. Macler and Merkle estimate that a significant fraction of water-borne disease outbreaks in the U.S. is associated with well water. In their paper they describe pathogenic microorganisms of concern for groundwater and methods for their control.

The effect of subsurface microbial processes on chemical reactions in aquifers has been the subject of a large body of research. Chapelle notes that microorganisms were first recognized as an agent in controlling groundwater chemistry around petroleum deposits in the early 1900's. He describes the importance of microorganisms in mediating reactions in aquifers that affect both geologic processes and human endeavors. The paper by Bennett and colleagues shows that microbially-driven reactions in aquifers can be due to either macroscale changes in aqueous chemistry or microscale perturbations around a single microorganism or colony. Four papers in this issue pertain to microbial degradation of subsurface contaminants. Haack and Bekins summarize observations of microbial populations in contaminant plumes. They discuss how variations in populations and associated degradative capabilities result from biological, chemical, and hydrologic factors. Lovley and Anderson provide an overview of the role of dissimilatory metal reduction on the fate of groundwater contaminants. They explain the importance of this process to natural attenuation of both organic and inorganic contaminants. Hoyle and Arthur comment that, although pesticides are increasingly being detected in groundwater, most studies have focussed on their fate in soils and the unsaturated zone. In their summary of the existing literature on biotransformation of pesticides below the water table, they note that subsurface physical and chemical heterogeneity may cause confusing and inconsistent results in biotransformation studies. Bradley describes the known biodegradation reactions of chlorinated ethenes and their implications for natural attenuation. He suggests that a number of newly documented anaerobic oxidation reactions may affect fate of chlorinated ethenes in groundwater under anaerobic conditions.

Many of the papers in this issue describe how physical and chemical variations in the subsurface result in changes in microbial populations. The implication for 
hydrogeologists is clear. Further insights into the subsurface as a habitat for microorganisms will require the understanding of subsurface properties provided by hydrogeologists. A traditional problem with interdisciplinary collaborations is one of educating collaborators on the methods and principles of another discipline. Thus, to facilitate continued collaborations between hydrogeologists and microbiologists, the final three papers in this special issue review methods used for subsurface microbial studies. Madsen's paper describes how nucleic-acid techniques can be used to characterize the identity and activities of subsurface microorganisms. He provides a useful summary of recent investigations of subsurface microorganisms using nucleic-acid procedures. Green and Scow provide an introduction to the use of phospholipid fatty acid analyses. In a comprehensive review of the literature from aquifer studies, they discuss the limitations and capabilities of this method. Finally, Murphy and Ginn review various mathematical expressions used to model microbial processes in porous media. In particular, the mechanisms by which microorganisms attach and detach from aquifer solids and are transported by groundwater is essential for understanding how new areas are colonized and for developing strategies to distribute microorganisms in contaminated subsurface systems. Mathematical expressions used to describe this process are reviewed and expanded on in their paper.

The influence of subsurface microorganisms is now recognized to range from groundwater quality to global chemical mass balances. The locations and activities of these organisms are controlled by subsurface chemical and physical properties that have traditionally been studied by hydrogeologists. As an illustration, consider two poorly understood issues in subsurface microbiology. These are the nature of subsurface microbial community interactions, and mechanisms by which viable microorganisms are transported in aquifers. Both of these processes are strongly affected by aquifer heterogeneity. Understanding them will require separating the fundamental microbial functions from the influences of aquifer properties. Thus, continued progress in this field will be founded on informed collaborations between hydrogeologists and microbiologists. As Guest Editor, I hope that this issue will help facilitate new and existing collaborations in this area.

Key words microbial processes - contamination · hydrochemistry $\cdot$ subsurface microbiology $\cdot$ bioremediation 